\title{
Distribution of particle sedimentation thickness under constant relative centrifugal force in rotating separation system using wireless electrical resistance detector
}

\author{
Yosephus Ardean Kurnianto PRAYITNO****, Tong ZHAO***, Yoshiyuki ISO**** and Masahiro TAKEI* \\ *Department of Mechanical Engineering, Chiba University, 1-33, Yayoicho, Inage-ku, Chiba-shi, Chiba 263-8522, Japan \\ E-mail: masa2@chiba-u.jp \\ **Mechanical Engineering Department, Vocational College, Universitas Gadjah Mada, Bulaksumur, Yogyakarta 55281, Indonesia \\ ${ }^{* * *}$ School of Mechanical and Precision Instrument Engineering, Xi'an University of Technology, Xi'an, Shaanxi 710048, China \\ ${ }^{* * * *}$ Heat and Fluid Dynamics G, Technology Platform Center, Technology \& Intelligence Integration IHI Corporation, 1, Shin-Nakahara- \\ Cho, Isogo-ku, Yokohama 235-8501, Japan
}

Received: 29 October 2019; Revised: 14 March 2020; Accepted: 25 May 2020

\begin{abstract}
The demand for high separation efficiency needs an advanced device that can be installed in the separation machinery. Parameters in the separation system, such as feeding flow rate and rotational speed, influence the sedimentation formation. A wireless electrical resistance detector (WERD) was developed with an ability to detect the change of the electrical properties of the suspension inside the centrifugal separation domain. The main focus is to observe the particle sedimentation thickness in the specific positions inside an industrial-scale centrifuge. This wireless instrument has an excellent performance in ultra-high rotation operating speed due to its lightweight and flexibility. WERD transmitted the measured resistance to the processing PC, which then processed the data using the particle thickness formula. The code refers to the resistance strength and normalization method under the boundary of the particle volume fraction in the sedimentation state. Simulation and experimental studies solved the constant of the particle thickness formula. In these studies, the sediment layer was represented by the suspended microsphere acrylic particles with a diameter of $10 \mu \mathrm{m}$. The suspension was a mixture of the aqueous Sodium Chloride and the acrylic particles. The real-scale experiment was conducted on industrial centrifuge with a constant relative centrifugal force of $R C F=2,130 \mathrm{G}$. As a result, the distribution of particle sedimentation thickness during the centrifugation was successfully observed by WERD. The results showed as the feeding rate increases; the particle sedimentation thickness increased up to near the feeding point. Under constant relative centrifugal force, the particle distribution rate under a low feeding flow rate gave a smoother thickening distribution. In the high feeding flow rate, the distribution was thicker and faster especially at the position nearer to the feeding point. These findings of the WERD application is useful for the non-invasive sedimentation monitoring in the separation system.
\end{abstract}

Keywords : Sedimentation, Separation, Wireless, Electrical resistance, Centrifugation

\section{Introduction}

Rotating separation systems, such as decanter centrifuge, are often utilized in the industrial field under extreme operating conditions. Separation problems occurred in particle separation in several areas such as chemical and food processing, wastewater treatment, pharmaceutical processes biotechnology, among others. In the past decade, successful research on the measurement of separation processes resulted in excellent acceptance in the academic and industrial fields. However, the on-line analysis of the separation process has not been clarified yet. An on-line measurement requires a device that can be attached and directly measure the separation process on centrifuges. The feasibility study on in-situ 
measurement produced good results quantitatively and could be a useful tool for on-line monitoring systems (Aoki et al., 2015). The in-situ concept supports the idea of developing a wireless measurement device for high-speed rotating separation systems.

The separation process can be measured by identifying the significant difference between the properties of two-phase particle-liquid. The distribution of the conductivity possesses a considerable aspect in determining the two-phase boundaries in the separation regime. Currently, the development of measurement techniques in process tomography is able to determine the electrical properties of its object varies greatly (Lutran et al., 1991), (Gladden et al., 2003), (Boyer et al., 2005), (Wang et al., 2013), (Zhao et al., 2015), (Tan et al., 2016). One of the appealing techniques is electrical resistance tomography (ERT). ERT has a superior temporal resolution ability even though it only images a cross-section area. ERT is applied variously such as in oil-water two-phase flow patterns measurement in horizontal pipes (Tan et al., 2016), in proper orthogonal flow regimes measurement in horizontal pipelines (Wang et al., 2013) and liquid distribution measurement in a trickle bed reactor (Zhao et al., 2015). ERT is, therefore, an effortless and robust technique with extensive applications. Two essential parameters desired in the centrifuge are rapid performance and non-invasiveness. ERT achieves the above desirable qualities with low power consumption during the imaging of specimens (Hallaji et al., 2015) even though ERT has a low spatial resolution.

Based on the advantages mentioned of ERT, an advanced real-time and in-situ separation detection device, the wireless electrical resistance detector (WERD) was proposed and validated experimentally. This device is the base model of the further developed device that applies the ERT technique in imaging the physical phenomenon inside the centrifuge. The real-time measured resistance data is wirelessly transmitted to a processing PC to show the distribution of the local particle sedimentation thickness. The WERD measures the particle sedimentation thickness as the reference parameter to comprehend the separation efficiency. Consequently, by controlling the rotation speed of the separation system and the feeding volumetric flow rate of the suspension, we can achieve the optimum setting for the industrial centrifuge.

\section{Nomenclature}

\begin{tabular}{|c|c|}
\hline$A$ & : Constant value referring to the range of the measured resistance based on the effective measuring area \\
\hline$B$ & : Einstein coefficient \\
\hline$c_{w}$ & : Weight percent of solid concentration in the suspension \\
\hline$D$ & : Diameter of the centrifuge bowl in a non-dimensional unit of $\mathrm{D}$ \\
\hline$D_{\text {pipe }}$ & : Diameter of the feeding point in a non-dimensional unit of $\mathrm{D}$ \\
\hline$E$ & : Electric field intensity \\
\hline$f$ & : Measurement frequency of WERD \\
\hline$h_{l}$ & : Heigth of debris layer \\
\hline$J$ & : Current density \\
\hline$k$ & : Non-similarity fitting constant \\
\hline$d L_{E}$ & : Arc-length in inspected media \\
\hline$l_{\mathrm{p}}$ & : Particle sedimentation thickness \\
\hline$n$ & : Normal vector \\
\hline$Q$ & : Volumetric feeding flow rate \\
\hline$R C F$ & : Relative centrifugal force \\
\hline$R e_{1}$ & : Low feeding flow rate inside the supply pipe \\
\hline$R e_{2}$ & : High feeding flow rate inside the supply pipe \\
\hline$R_{\mathrm{N}}$ & : Normalized resistance \\
\hline$R_{\mathrm{N}(\mathrm{lp} \text { Max })}$ & : Normalized resistance of the upper bound; refers to the closest-pack resistance \\
\hline$R_{\text {ref }}$ & : Reference resistance of liquid phase \\
\hline$R_{\mathrm{xi}}$ & : Measured resistance correspond to particle sedimentation thickness \\
\hline$t$ & : Duration of the WERD measurement \\
\hline ts & : Time step of the WERD measurement \\
\hline
\end{tabular}




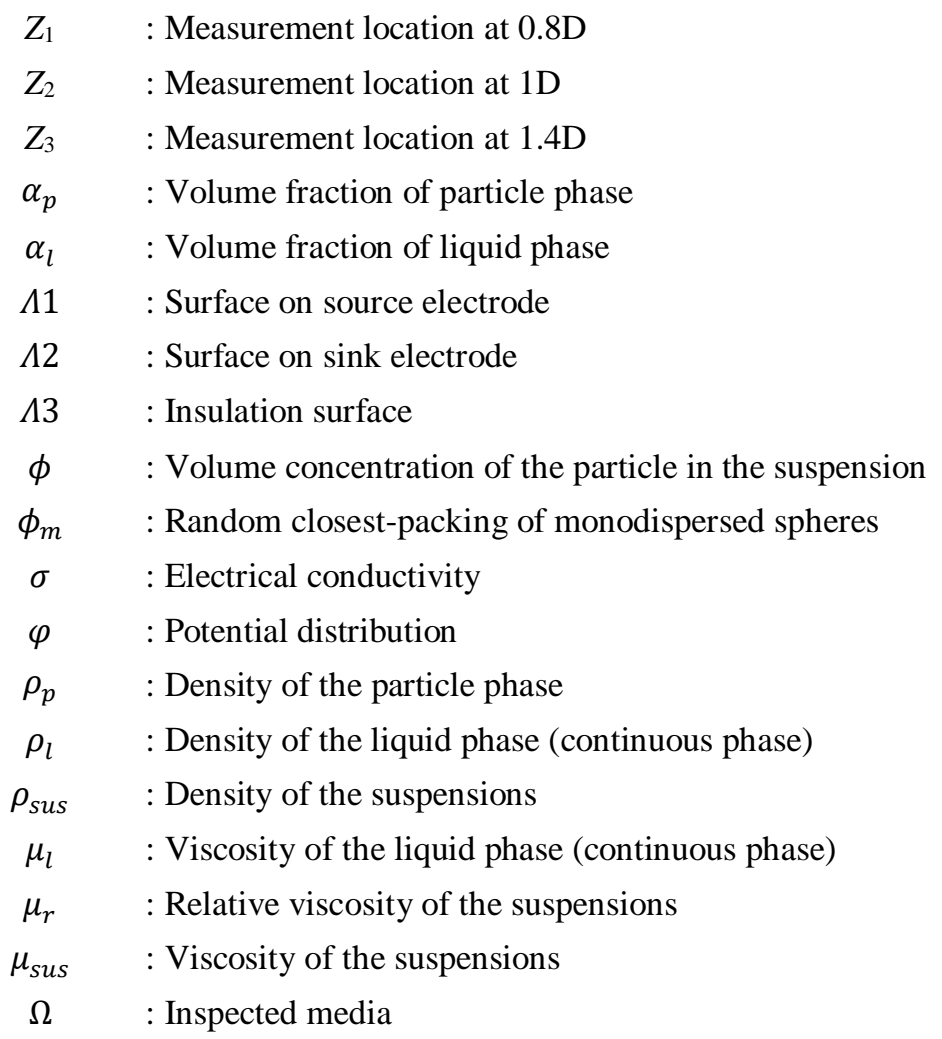

\section{Wireless Electrical Resistance Detector (WERD)}

\subsection{The system principle of WERD}

Figure 1 shows the innovative concept and the system diagram of the WERD measurement device on the industrial centrifugal. WERD introduces three parts that work-integrated on the separation system to the monitoring PC. WERD consists of detection, communication, and power supply parts. In the detection part, the electrodes are attached to the rotating bowl of the centrifuge. These detectors are connected to the primary system of WERD includes the microcomputer, the signal generator, and the measurement circuit. The signal generator is known as an AC generation device (AGD) that injects the constant current to the electrodes. The voltage measurement circuit (VMC) is then sending the measured voltage to the wireless transmission module (WTM) and wirelessly transmits the data. A processing unit receives and processes the measured resistance. In this system, the microcomputers (PIC16F88) control all procedures of the measurements. An algorithm of the conversion formula predicts the sedimentation concerning the liquid and particle electrical properties. Therefore, the particle sedimentation thickness could be quantitatively observed. Moreover, this system was successfully used to measure the particle volume fraction in centrifugal particles-liquid two-phase fields (Zhao et al., 2018), (Atagi et al., 2019).

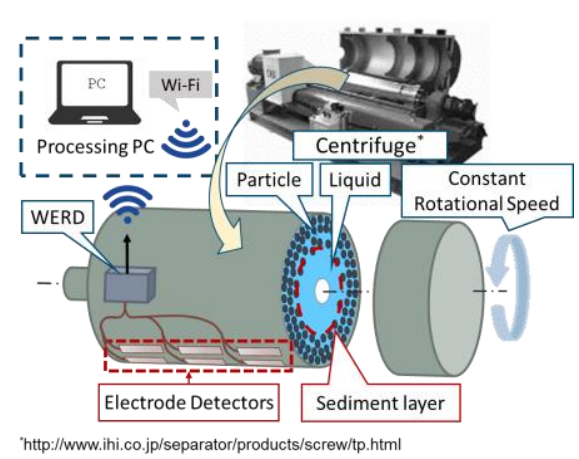

(a) Innovative concept of WERD

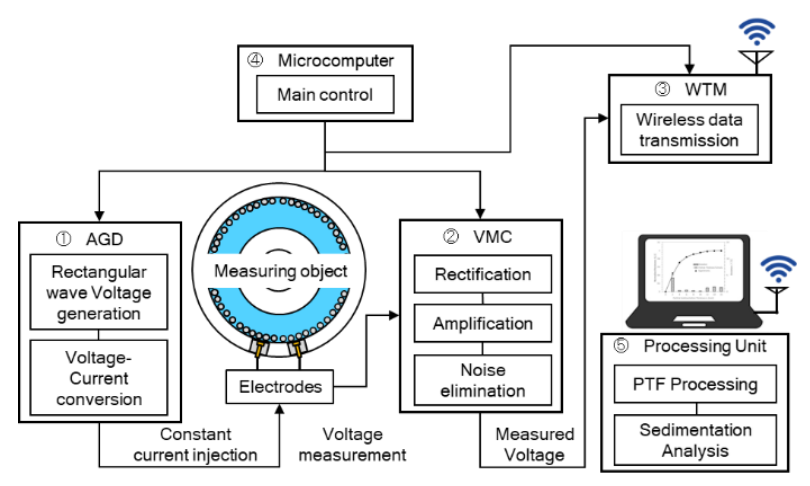

(b) System diagram of WERD

Fig. 1 The system principle of WERD on industrial centrifugal. 
The measurement of WERD is based on the principle of electrical resistance of the pole to pole array. WERD system measures the voltage difference due to the conductivity change as the detection of the particle presence inside the centrifuge. Since WERD applies a two-adjacent electrode, the measurement domain is limited to the arc-length inspected media above the electrodes. In this domain, the conductive and non-conductive conditions refer to the presence of liquid and particle-phase inside the centrifuge, respectively. Assuming the inspected media is in a quasi-static approximation, the electric fields will change simultaneously and synchronously in response to the AC constant current excitation by the signal generator. Here, the inspected media acts in zero charge density; when there is no current resource or current influx that results in the divergence of current is null. This condition is met due to the effect of no excitation resource and its vortex within the inspected media of ERT (Wang et al., 2013). Therefore, any spot in the inspected media is explained as follows:

$$
\begin{aligned}
& \nabla \cdot J=0\left\{\begin{array}{c}
J=\left(\sigma+j \omega \varepsilon_{0} \varepsilon_{r}\right) \cdot E \\
E=-\nabla \varphi
\end{array}\right. \\
& \nabla \cdot(\sigma \cdot \nabla \varphi)=0 \text { in } \Omega
\end{aligned}
$$

where $J$ is current density, $\sigma$ is electrical conductivity, $E$ is electric field intensity, $\varphi$ is potential distribution in the field, $\Omega$ is the inspected media, $\varepsilon_{0}$ is the permittivity of vacuum, $\varepsilon_{r}$ is the relative permittivity $\varepsilon_{r}$. Since the twophase objects have different conductivities, the potential distribution $\varphi$ in Eq. (2) under the boundary condition of input and output current between the electrode detectors also the insulation of in a wall, as defined in Eq. (3).

$$
\int_{\Lambda i} \frac{\partial \varphi}{\partial n} \cdot d L_{E}=\left\{\begin{array}{c}
+i \\
-i \\
0
\end{array} \text { on } \partial \Omega \text { for } i=1,2,3\right.
$$

where $\Lambda 1, \Lambda 2$, and $\Lambda 3$ are the surface on the source electrode, sink electrode, and in the insulation surface of the electrode, respectively, while $n$ is the outward normal vector of the boundary.

\subsection{Development of particle thickness formula (PTF)}

Particle sedimentation affects the measurement of WERD due to the presence of lower conductance distribution in the inspected media. The thickness of sedimentation is measured by observing the correlation between the electrical parameter of resistance into the physical parameter of the particle thickness formula (PTF). PTF introduces the conversion equation to determine the particle sedimentation thickness based on the measured resistance. The development method of PTF considers two critical information on the whole measurement domain of the liquid and solid fraction. The specific domain in the inspected media is needed to give an accurate detection of the particle presence inside the centrifuge. This domain represents the optimum measured area in terms of measured resistance data. A normalization method is applied, which has been widely used in the processing method in the electrical resistance technique. In this study, the normalization method based on the reference value that refers to the measured data of aqueous Sodium $\mathrm{Chloride}_{(\mathrm{NaCl}} \mathrm{N}_{(\mathrm{l})}$ ) Thus, the reference value subtracts the measured resistance results in a new value. The maximum of this new value divides the measured data gained from the experimental works to proceed with the normalization as a non-dimensional unit. The normalized measured resistance $R_{\mathrm{N}}$ is solved in Eq. (4). Since the resistance value has a physical meaning of the particle sedimentation thickness, an approach based on the non-linear fitting of resistance strength is used (Eq. (5)). This approach accommodates the distribution of normalized measured resistance to calculate the particle sedimentation thickness with a high fitting level.

$$
\begin{aligned}
& R_{\mathrm{N}}=\frac{R_{\mathrm{xi}}-R_{\mathrm{ref}}}{A} ; \text { where } A=R_{\mathrm{x}\left(l_{\mathrm{p}} \text { Max }\right)}-R_{\mathrm{ref}} \\
& R_{\mathrm{N}}=R_{\mathrm{N}\left(l_{\mathrm{p}} \text { Max }\right)}\left(1-e^{\frac{-l_{\mathrm{P}}}{k}}\right)
\end{aligned}
$$

where $k$ is the non-linear constant value that refers to the electrode characteristics, $A$ is the constant value that refers to the range of the maximum measured resistance based on the effective measuring area, $R_{\mathrm{xi}}$ is the corresponding resistance to the specific thickness measurement, $R_{\mathrm{x}(\mathrm{lp} \text { Max })}$ is the measured resistance at a maximum-possible thickness in the effective measuring area, and $R_{\text {ref }}$ is the reference resistance of the liquid phase only. The effective measuring area 
represents the possible thickness to be measured in the whole measurement domain (Zhao et al., 2018). Therefore, the constant $A$ was derived from the measured resistance of the maximum-possible thickness in this effective measuring area. Considering the actual data of the sediment thickness that possibly occurred during the centrifugation, we observed the change of the electric properties at the maximum thickness of $l_{\mathrm{p}}=15 \mathrm{~mm}$.

In the high-rotational speed machines, the operational condition and settings play an essential role in the result of the separation products. The operational condition during the separation process applies three separation factors, such as the filter media for filtering, sludge layer thickness, and separation force on the sludge affecting the sludge dewaterability (Hiratsuka and Kyu, 1991). An on-site separation system showed that the dewaterability of waste excess sludge was very poor, and the efficient solid-liquid separation was considered to be demanding without any other pre-treatment to the sludge (Ogawa et al., 1996). Thus, the in-situ measurement considering the separation factors is essential. Here, we introduce PTF derived from the measured resistance data of WERD as a similar factor as the sediment layer thickness. Since the term of the sludge layer thickness corresponds to the separated solid phase from the suspension in the separation system, the particle thickness formula of WERD limit the detection of the sediment layer only at the closest-pack particle volume fraction. This approach considers the occurred sedimentation under a continuous feed during the centrifugation. Moreover, following Cao et al. (2015) works, we assumed the settling of the large particle in the dilute suspension has little influence on the settling rate of the interface between the dispersion and clear liquid.

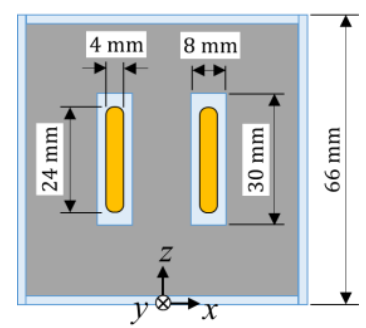

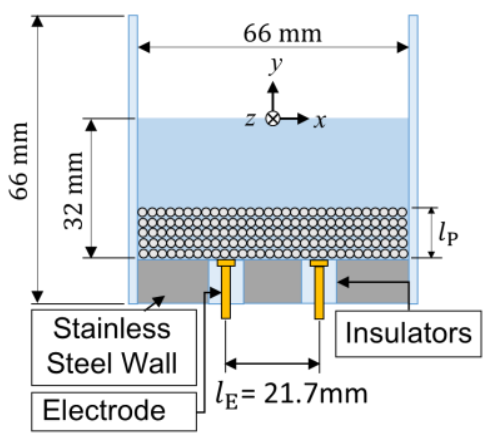

(a) Cubical apparatus

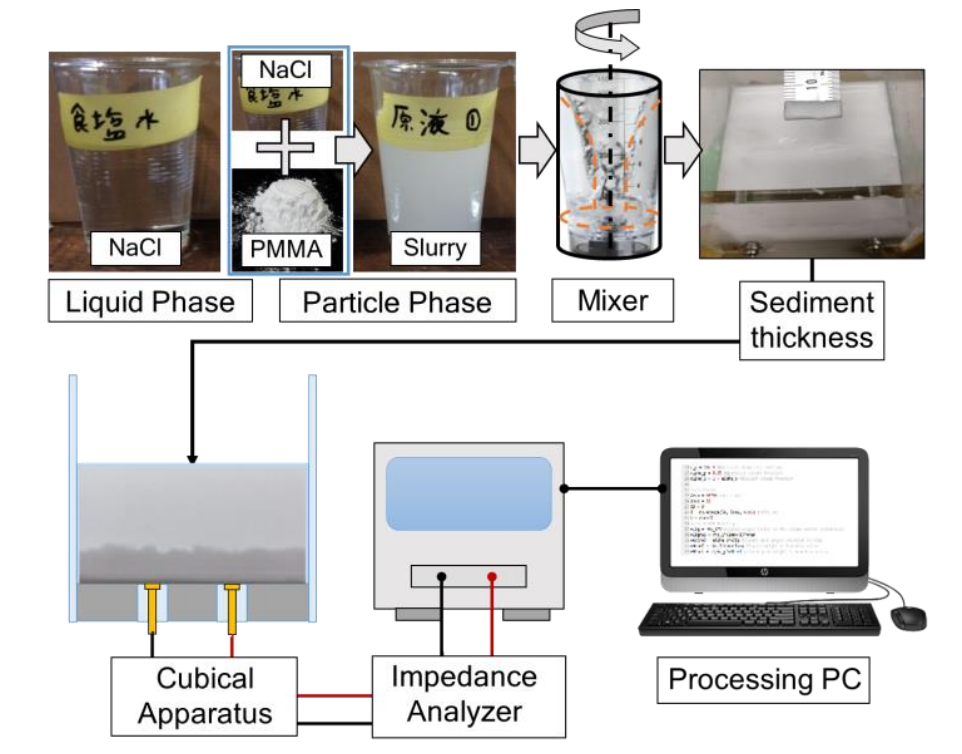

(b) Experimental setup

Fig. 2 Schematic of the experimental setup using two-adjacent electrodes of cubical apparatus.

\subsection{Static experimental and simulation studies}

We modeled static particle sedimentation in a cubical apparatus with dimensions of length $l \times$ width $w \times$ height $h=$ $66 \times 66 \times 66 \mathrm{~mm}^{3}$. Two-adjacent electrodes were insulated by an acrylic resin with the dimensions of length $l \times$ width $w$ x height $h=24 \times 4 \times 1 \mathrm{~mm}^{3}$ and $30 \times 8 \times 1 \mathrm{~mm}^{3}$, respectively. The main body of the apparatus is made of transparent acrylic and equipped with a stainless steel bottom. The electrode material, stainless steel, was chosen based on the material selection of the industrial centrifuge considering a similar operating condition in the real-scale application. The insulator in the electrodes prevents short-circuiting and provides null electrical excitation to the wall. The cubical apparatus and electrode diagrams are shown in Figure 2 (a). The research objects are an aqueous Sodium Chloride $\left(\mathrm{NaCl}_{(1)}\right) 1.5 \mathrm{wt} . \%$ as a liquid phase and a sediment phase of $10 \mu \mathrm{m}$ microspheres $\mathrm{PMMA}_{(\mathrm{s})} / \mathrm{NaCl}_{(\mathrm{l})} 1.5 \mathrm{wt}$.\%. This sediment is the main object that formed the sediment layer by using the industrial centrifuge. Here, the sediment layer refers to the assumption of the closest-packing of the particle volume fraction in the liquid by $\alpha_{p}: \alpha_{l}=0.65: 0.35$. The height of the debris layer is $h_{l}=32 \mathrm{~mm}$, referring to the height of the liquid phase remaining inside the industrial centrifuge. We used an impedance analyzer with a constant current of $J=0.1 \mathrm{~mA}$ to measure the voltage difference inside the inspected media. 
The model of the particle thickness is implemented in a $1 \mathrm{~mm}$ accuracy order with a data matrix of $l_{\mathrm{p}}=1,2, \ldots, 15 \mathrm{~mm}$ refers to the maximum possible thickness during separation in the industrial centrifuge. In this experiment, we assumed the sedimentation thickness is in a static condition. First, we prepared the sediment material by mixing the particle and liquid using a 2,000 rpm mixer within 1 minute. Then, we formed the sedimentation thickness on the bottom of the cubical apparatus following the data matrix of $l_{\mathrm{p}}$. The liquid phase is then gently supplied into the cubical apparatus to the debris height of $h_{l}=32 \mathrm{~mm}$. Finally, the resistance data derived from the voltage difference between the two adjacent electrodes is measured using the impedance analyzer (Hioki E.E.Corp) (Figure 2 (b)).

Particle thickness formula on Eq. (5) gives a correlation to the particle sedimentation thickness $l_{\mathrm{P}}$ for each normalized resistance measured $R_{\mathrm{N}}$. This method provides the distribution of particle sedimentation thickness based on the limitation given by Eq. (4). A simulation study on the effective measurement domain of the electric field is conducted using a multiphysics software COMSOL ${ }^{\circledR}$ to clarify the static experimental results. The simulation setup is set in a similar condition to the experimental works with a simplification on the static-condition. We simulated a three-dimensional model of the cubical apparatus with a physic case on electric currents (ec). We built two models, cubical model and centrifuge model, to clarify the effects of the difference surface of the electrodes. The dimension of these models refer to the dimensions explained in section 3.2. The preset study was in the frequency domain with a setting in the single frequency of $f=2 \mathrm{kHz}$ referring to the applied frequency of the WERD. The governing equation in the frequency domain refers to Eq. (1) and (2). The current density is affected by the electromagnetic constant of vacuum permittivity $\varepsilon_{o}=$ $8.854 \cdot 10^{-12} \mathrm{~F} / \mathrm{m}$. The different electrical properties of the measured objects are inputted as the electrical conductivity $\sigma$ and the relative permittivity $\varepsilon_{r}$. The applied constant current in WERD was used in the simulation as the current source with a value of $I c=1 \mathrm{~mA}$. In order to model the sediment thickness, we assume a homogenous state of sedimentation in the simulation. The electrical properties of the separation media are shown in Table 1.

Table 1 Electrical properties of measured objects and electrodes.

\begin{tabular}{|l|c|c|c|}
\hline \hline \multicolumn{1}{|c|}{ Physical properties } & $\begin{array}{c}\text { Liquid: } \\
\mathrm{NaCl}_{(\mathrm{l})}\end{array}$ & $\begin{array}{c}\text { Sediment: } \\
\mathrm{PMMA}_{(\mathrm{s})} / \mathrm{NaCl}_{(\mathrm{l})}\end{array}$ & $\begin{array}{c}\text { Electrodes: } \\
\text { Stainless Steel }\end{array}$ \\
\hline Electrical conductivity $\sigma[\mathrm{S} / \mathrm{m}]$ & 4.25 & 1.49 & $1.47 \times 10^{6}$ \\
Relative permittivity $\varepsilon_{r}[-]$ & 74.00 & 27.85 & $1.00 \times 10^{-15}$ \\
\hline
\end{tabular}

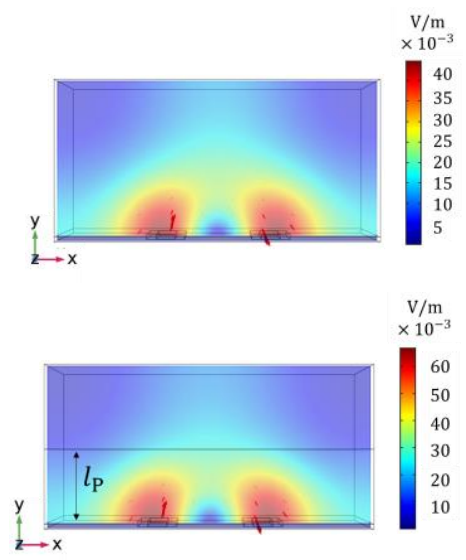

(a) Cubical model

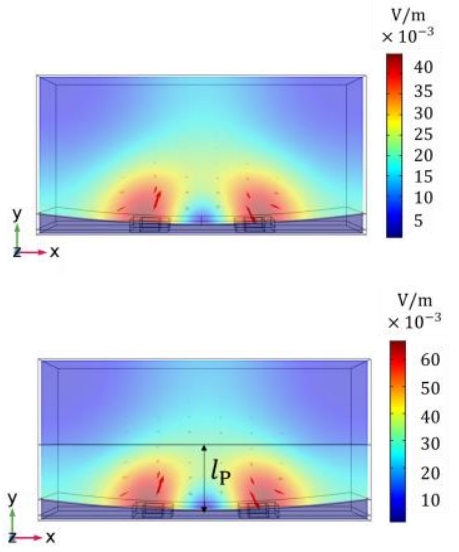

(b) Centrifuge model

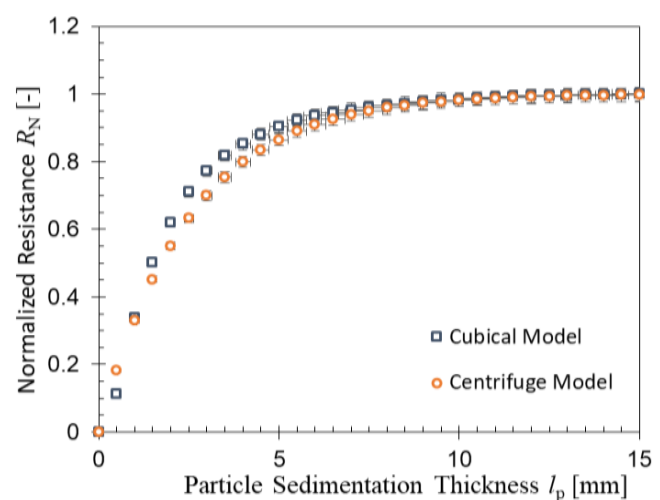

(c) Comparison between the two models

Fig. 3 Electric field of the cubical and centrifuge model by simulation study.

Figure 3 shows the electric field of the cubical and centrifuge model in two conditions, liquid only and the maximumpossible thickness. The electrical property of the real liquid used in the experiment is set as a reference in the simulation setup. This reference is determined as the lower bound in the normalization method. For the upper bound, the maximumpossible thickness occurred is simulated. The electric field between two models of cubical apparatus and real centrifuge is observed in slightly different due to the different electrodes angle (Figure 3 (c)). The real scale centrifuge has curved- 
surface electrodes but in a similar dimension to the planar-surface electrodes of the cubical model. The centrifuge model demonstrated a more focused electric field in the center or between the two-adjacent electrodes (Figure 3 (b)). This factor is vital in developing the non-linear constant $k$.

As a result, both works present the distribution of the measured resistance as a function of the particle sedimentation thickness. The simulation and experimental works demonstrate a distribution of the electric field on the variation of the particle sedimentation thickness, respectively. Based on the distribution of the electric field inside the centrifuge model, the simulation works confirmed that the maximum-possible thickness is at $l_{\mathrm{p}}=15 \mathrm{~mm}$. This limitation also confirmed the effective range in measuring the voltage change for the WERD system. The measured resistance from simulation and experimental works were normalized using Eq. (5). These two normalized measured resistances are compared to produce the non-linear constant $k$ to develop the PTF. In Eq. (5), the maximum normalized resistance is set as $R_{\mathrm{N}\left(l_{\mathrm{p}} \mathrm{Max}\right)}=1$ to define the measurement of the object reference on the PTF. A high degree of the fitting is desired to ensure the best accuracy of the particle sedimentation thickness, which is accommodated using the resistance strength correlation and solved by Eq. (6).

$$
l_{\mathrm{P}}=-2.5 \cdot \ln \left(1-R_{\mathrm{N}}\right)
$$

where $l_{\mathrm{p}}$ is the particle sedimentation thickness with non-linear constant $k=-2.5$, and $R_{\mathrm{N}}$ is the normalized measured resistance. The correlation between the measured resistance value and the particle sedimentation thickness $l_{\mathrm{p}}$ is shown in Figure 4. The particle thickness formula has an excellent agreement to both works, simulation, and experiment, with an average deviation of $1.61 \%$.

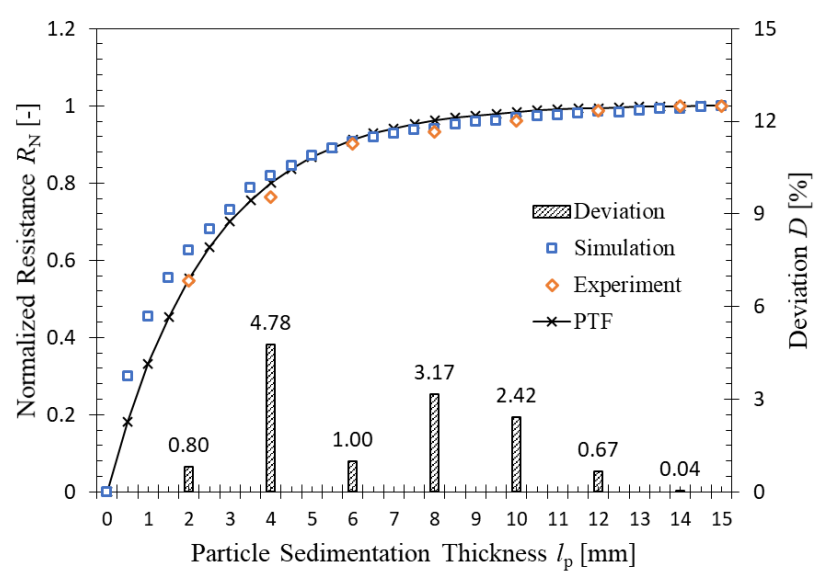

Fig. 4 Distribution of particle sedimentation thickness using PTF compared with experiment and simulation results.

\section{Real-scale experiment \\ 4.1. Experimental setup}

A real centrifuge was used in this experimental study, as shown in Figure 5. The centrifuge was equipped by electrode detectors, which were covered with the dielectric material of acrylic. This set of detectors was taped into the inner wall of the cylindrical bowl of the centrifuge. We used similar electrodes and its insulator with the dimensions of length $l \mathrm{x}$ width $w \times$ height $h=24 \times 4 \times 1 \mathrm{~mm}^{3}$ and $30 \times 8 \times 1 \mathrm{~mm}^{3}$, respectively. The detectors were taped in the specific location of the centrifuge as following $Z_{1}=0.8 \mathrm{D}, Z_{2}=1 \mathrm{D}, Z_{3}=1.4 \mathrm{D}$ with $\mathrm{D}$ refers to the diameter of the bowl. This measurement was in simultaneous assessment by using $\mathrm{I}_{2} \mathrm{C}$ that communicated wirelessly to a processing unit near the centrifuge. In the feeding point, suspension of $\mathrm{PMMA}_{(\mathrm{s})} / \mathrm{NaCl}_{(\mathrm{l})}$ was constantly-supplied into the inside bowl to conduct the separation process. 


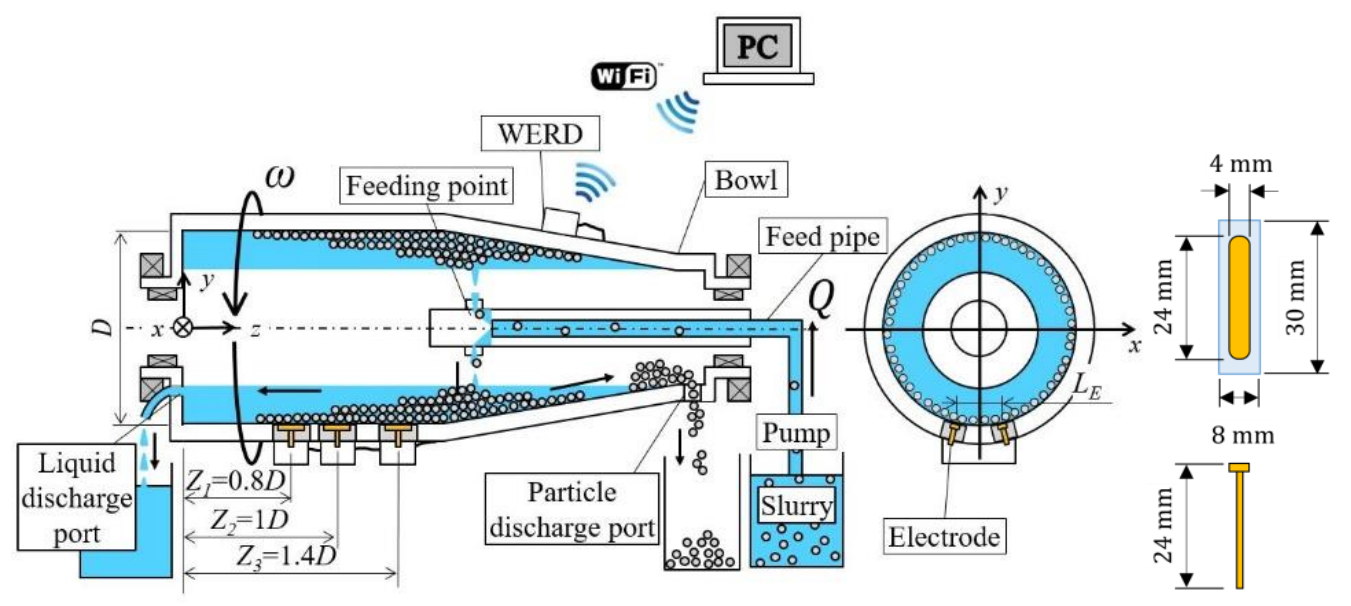

Fig. 5 Schematic diagram of the industrial centrifuge experimental setup.

\subsection{Experimental methods and conditions}

The liquid phase was the aqueous Sodium Chloride $\left(\mathrm{NaCl}_{(1)}\right) 1.5 \mathrm{wt} . \%$ and the particle phase was the PMMA with 1 wt. \%. The suspension density considers the concentration of solids by weight in the suspension, the density of the particle phase, and the density of the liquid phase. The density of the suspensions is defined in Eq. 7.

$$
\rho_{\text {sus }}=\frac{100}{\left(\frac{c_{w}}{\rho_{\mathrm{p}}}+\frac{\left(100-c_{w}\right)}{\rho_{\mathrm{l}}}\right)}
$$

where $c_{w}$ is the weight percent of solid concentration in the suspension with the value of $c_{w}=1 \%, \rho_{\mathrm{p}}$ is the density of the particle phase with the value of $\rho_{\mathrm{p}}=1,190 \mathrm{~kg} / \mathrm{m}^{3}$, and $\rho_{1}$ is the density of the liquid phase (continuous phase) with the value of $\rho_{1}=1,008 \mathrm{~kg} / \mathrm{m}^{3}$. Hence, the density of the suspension is obtained as $\rho_{\text {sus }}=1,009 \mathrm{~kg} / \mathrm{m}^{3}$.

The suspension viscosity is calculated by considering the relative viscosity. Krieger and Dougherty (1959) observed the relationship between the successive packets of suspension to the total particle volume fraction and to the suspension viscosity to construct the relative viscosity. Since the viscosity of suspension is the amplification of the liquid viscosity (continuous phase) as $\mu_{\text {sus }}=\mu_{\mathrm{r}} \times \mu_{\mathrm{l}}$, Eq. 8 defines the viscosity of suspension:

$$
\mu_{\text {sus }}=\mu_{l}\left(1-\frac{\phi}{\phi_{m}}\right)^{-B \phi_{m}}
$$

where $\mu_{1}$ is the liquid (continuous phase) viscosity with the value of $\mu_{1}=1.023 \mathrm{mPa} \cdot \mathrm{s}, B$ is the Einstein coefficient with the value of $B=2.5, \phi$ is the particle in the suspension volume fraction with the value of $\phi=0.01$, and $\phi_{m}$ is the random close-packing of monodispersed spheres with the value of $\phi_{m}=0.64$ (Rintoul and Torquato, 1996). Hence, the viscosity of the suspension is obtained as $\mu_{\text {sus }}=1.049 \mathrm{mPa} \cdot \mathrm{s}$. Based on these properties, the feeding flow rate flows through a feeding point on a pipe with an inner diameter of $D_{\text {pipe }}=0.03 \mathrm{~m}$ is presented in two variation of Reynolds number, $R e_{1}=2,837$ and $R e_{2}=8,510$. In this experiment, measurements were performed at different positions $Z_{\mathrm{n}}$ under constant rotational speed. Experiments were carried out under the relative centrifugal force of $R C F=2,130 \mathrm{G}$.

The measurement was focused on three measurement locations, as explained in section 4.1. The method is defined as follows. First, after conditioning the rotational speed of the centrifuge, the liquid phase was injected inside the bowl. The inner vicinity of the bowl will be filled by this solution in an amount of time to reach a steady-state. Afterward, measurement by WERD is started, and subsequently, the suspensions were injected and continuously supplied for 10 minutes while the measurement of the resistance is simultaneously performed. The processing unit was located near the centrifuge to receive the transmitted data from the communication part of WERD. The process is ended by stopping the supply of the suspension, and the rotational speed is reduced to zero.

\section{Results and discussions}

WERD measured the separation process sequentially, as explained in section 4.2. Aqueous sodium chloride was first supplied into the bowl and measured by WERD. The liquid resistance became the reference parameter in Eq. (4) to calculate the normalized resistance of the sediment layer. The sedimentation stage was determined in the range of the 
time steps $t s$ with the total duration of $t=10$ minutes. Figure 6 (a) shows the particle sedimentation thickness at measuring the position of $Z_{1}=0.8 \mathrm{D}$ under low feeding flow rate $R e_{1}=2,837$. The distribution of particle sedimentation thickness $l_{\mathrm{p}}$ has been successfully obtained applying Eq. (6). At this position, the particle sedimentation thickness was measured in an uptrend, but under the final average thickness of $l_{\mathrm{p}}=2.5 \mathrm{~mm}$ (Figure 6 (a)). The final average thickness corresponds to the last thickness that occurred after the centrifugation. Based on the distribution under $R e_{1}=2,837$, the presence of the sediment layer at $Z_{1}=0.8 \mathrm{D}$ is limited. According to the centrifuge design (Figure 5), this location was the closest position to the liquid discharge port as of dominantly occupied by the liquid phase. Figure 6 (b) shows the particle sedimentation thickness at measuring the position of $Z_{2}=1 \mathrm{D}$ under the same feeding flow rate, $R e_{1}=2,837$. It can be seen that the presence of the particle is increased. The particle sedimentation thickness at $Z_{2}=1 \mathrm{D}$ is higher than at $Z_{1}=$ $0.8 \mathrm{D}$. Beyond the time step of $t s=8$, the sediment thickness was measured higher than $l_{\mathrm{p}}=2.1 \mathrm{~mm}$, which is the maximum thickness that occurred at $Z_{1}=0.8 \mathrm{D}$. It indicates that the particle was settled much higher. The particle distribution from the feeding point to this position is a half distance to the liquid discharge port, which means the percentage of the liquid and solid phase is assumed to be at 50:50. Figure 6 (c) shows particle sedimentation thickness at $Z_{3}=1.4 \mathrm{D}$, the closest position to the feeding point, under a low feeding flow rate $R e_{1}=2,837$. The distribution of particle sedimentation thickness $l_{\mathrm{p}}$ is higher than at the other measuring locations. Especially in the time step above $t s=6$, the thickness is measured higher than at $Z_{2}=1 \mathrm{D}$, where the maximum sediment thickness only occurred in $l_{\mathrm{p}}=2.53 \mathrm{~mm}$. It indicates that the presence of the particle at this position is dominant.

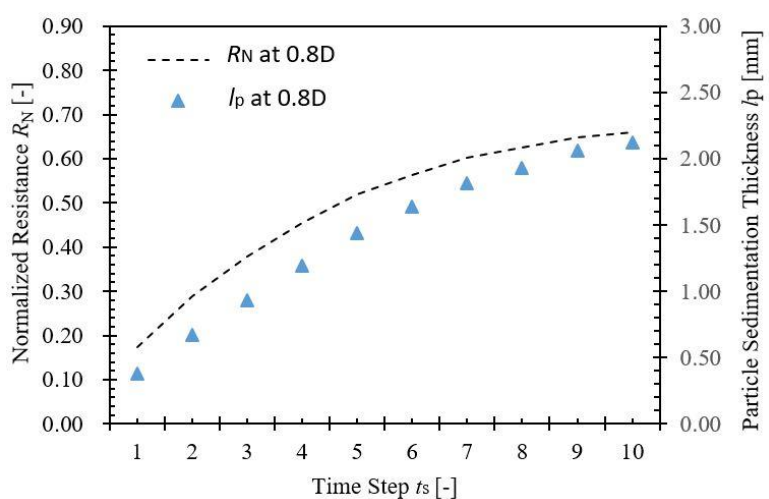

(a)

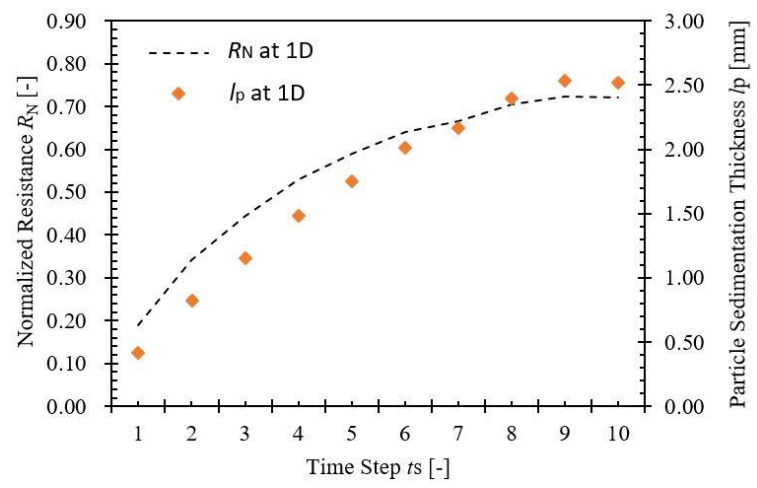

(b)

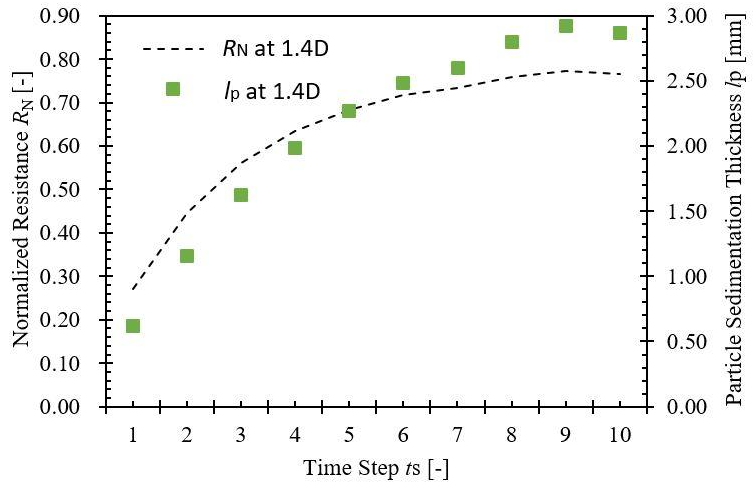

(c)

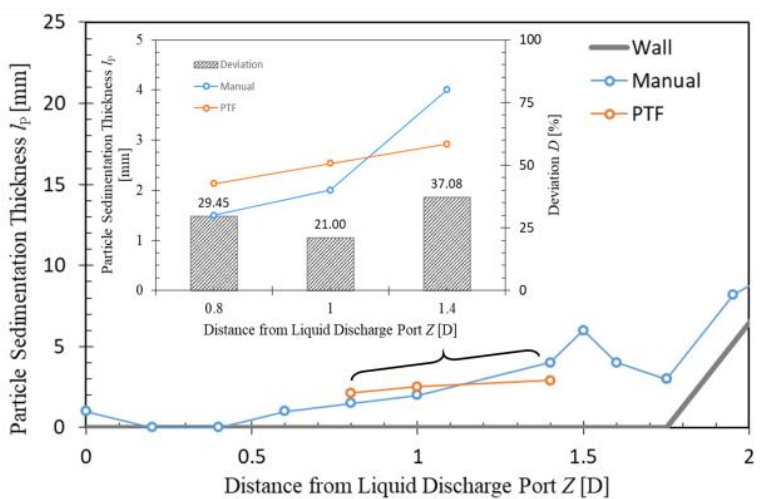

(d)

Fig. 6 Particle sedimentation thickness under low feeding flow rate $R e_{1}=2,837$ at (a) $Z_{1}=0.8 \mathrm{D}$, (a) $Z_{2}=1 \mathrm{D}$, (c) $Z_{3}=1.4 \mathrm{D}$, (d) Final thickness comparison between manual and PTF methods.

Figure 6 (d) explains the difference on the final sediment thickness between manual measurement and PTF methods. Manual measurement was done by using a centrifuge-shaped scale to measure the last thickness that occurred after the centrifugation manually. The particle sediment thickness by PTF at the end of the centrifugation time steps were then compared to this manual measurement. The final average thickness at all positions was $l_{\mathrm{p}}=2.5 \mathrm{~mm}$. It can be seen at the 
measurement point of $Z_{1}=0.8 \mathrm{D}$, and $Z_{2}=1 \mathrm{D}$, the tendency of the increasing sediment thickness was showed in the same uptrend. At the measurement point of $Z_{2}=1.4 \mathrm{D}$, the closest position to the feeding point, the manual measurement showed a significantly higher than the PTF results. In this position, the manual measurement gave a sediment thickness of $l_{\mathrm{p}}=4 \mathrm{~mm}$, while the sediment thickness of PTF only gave $l_{\mathrm{p}}=2.92 \mathrm{~mm}$. We assumed that during the shutdown period, some sediment layer was settled and formed an extra thickness. However, at the measurement point of $Z_{1}=0.8 \mathrm{D}$ and $Z_{2}$ $=1 \mathrm{D}$, the manual measurement gave lower sediment thicknesses than PTF results by deviations of $29.45 \%$ and $21 \%$, respectively. We assumed that the current PTF has a limitation on the fixed particle concentration for the sediment layer. As explained in section 3.2, the upper boundary of the normalization refers to the closest-pack, which is the sediment layer. This method limits the observation of the particle concentration in the sedimentation thickness. We bring up this issue in our next study by considering the reactance-resistance and phase-impedance correlation to reveal the particle concentration that changes during the separation process.

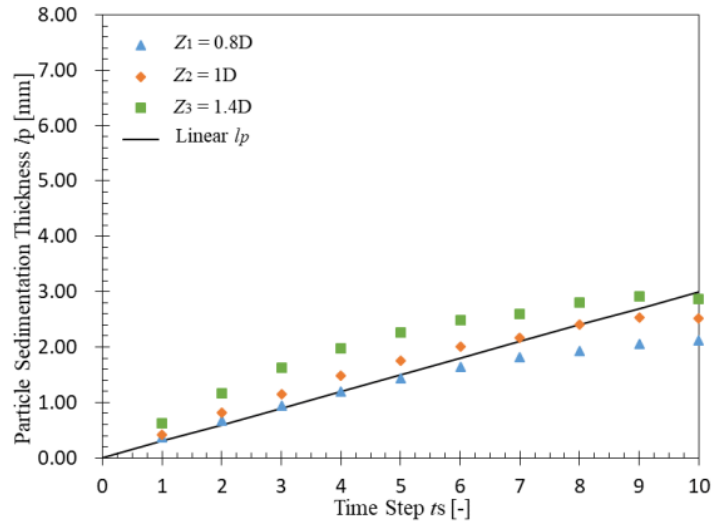

(a)

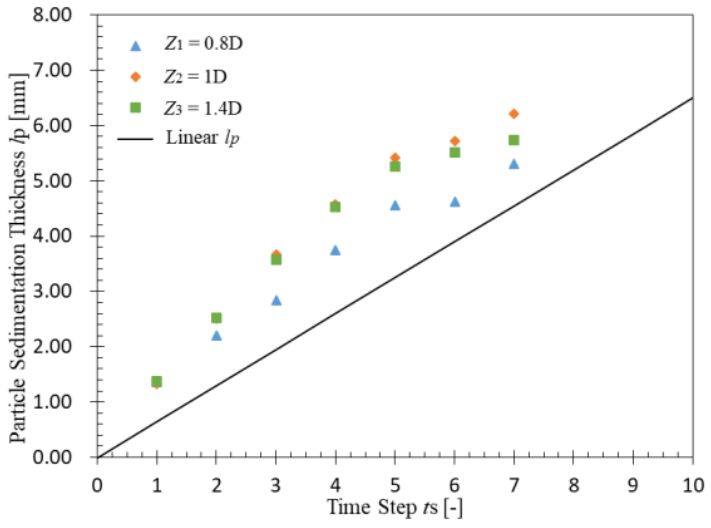

(b)

Fig. 7 Distribution of sedimentation thickness at various measurement locations under (a) low feeding flow rate $R e_{1}=2,837$; (b) high feeding flow rate $R e_{2}=8,510$.

Figure 7 shows the distribution of the particle sedimentation thickness at all measuring positions with the variation of feeding flow rates. The distribution was compared by the linear particle sedimentation thickness, which refers to the highest sediment thickness at $Z_{3}=1.4 \mathrm{D}$ for each feeding flow rate. The highest sediment thicknesses were set as $l_{\mathrm{p}}=3 \mathrm{~mm}$ for a low feeding flow rate and $l_{\mathrm{p}}=5 \mathrm{~mm}$ for a high feeding flow rate. It can be seen in Figure 7, the feeding flow rate significantly influences the particle sedimentation thickness inside the rotating centrifuge. Since the relative centrifugal force is constant, the feeding flow rate becomes the dominant factor in the separation process. The sedimentation under low feeding flow rate of $R e_{1}$ $=2,837$ gives a smoother thickening distribution following the handling of linear $l_{\mathrm{p}}$. On the contrary, under high feeding flow rate of $R e_{2}=8,510$, thicker particle sedimentation with a maximum value of $l_{\mathrm{p}}=6.2 \mathrm{~mm}$ is achieved. In Figure 7 (b), at the position of $Z_{1}=1 \mathrm{D}$ and $Z_{3}=1.4 \mathrm{D}$, the sedimentation was measured in almost equivalent thickness. It indicates the centrifugation has a similar effect in the area between these two positions. Compared to the lean distribution under low feeding flow rate, a higher relative centrifugal force could be needed for a high feeding flow rate of $\operatorname{Re}_{2}=8,510$ in order to give a better separation process. However, in this high feeding flow rate, some dispute data were observed beyond the time step of $t s=7$, which affects the measurement in the last time step could not be done.

\section{Conclusions}

In this research, we have clarified the separation process by electrical resistance measurement inside the centrifuge. A normalization method has been applied to explain the measured data of the sediment thickness inside the centrifuge. The particle thickness formula is developed by good agreement of experimental and simulation results based on the WERD system. Quantitative particle sedimentation thickness in the measuring positions inside the centrifuge was successfully detected. The results showed as the feeding rate increases; the particle sedimentation thickness increased up to near the feeding point. Under constant relative centrifugal force, the particle distribution rate under a low feeding flow rate gave a smoother thickening distribution. In the high feeding flow rate, the distribution was thicker and faster, 
especially at the position nearer to the feeding point. These findings give essential information to optimize the operational settings of the centrifuge. Moreover, the WERD application is useful for the non-invasive sedimentation monitoring in the separation system.

\section{Acknowledgment}

This work was supported in part by the IHI Corporation and the National Natural Science Foundation of China under Grant 51876175.

\section{References}

Aoki, S. and Ihara, I., Feasibility study on ultrasonic in-situ measurement of friction surface temperature, Mechanical Engineering Journal, Vol.2, No.1 (2015), DOI: 10.1299/mej.14-00431.

Atagi, Y., Zhao, T., Iso, Y. and Takei, M., Real-time imaging of particle distribution in centrifugal particles-liquid twophase fields by wireless electrical resistance tomography (WERT) system, IEEE Access, Vol.7 (2019), pp.1270512713.

Boyer, C., Koudil, A., Chen, P. and Dudukovic, M. P., Study of liquid spreading from a point source in a trickle bed via gamma-ray tomography and CFD simulation, Chemical Engineering Science, Vol.60, No.22 (2005), pp.6279-6288.

Cao, D., Iritani, E. and Katagiri, N., Solid-liquid separation properties in centrifugal sedimentation of bidisperse colloidal suspension, Journal of Chemical Engineering of Japan, Vol.48, No.7 (2015), pp.556-563.

Gladden, L. F., Lim, M. H. M., Mantle, M. D., Sederman, A. J. and Stitt, E. H., MRI visualization of two-phase flow in structured supports and trickle-bed reactors, Catalysis Today, Vol.79-80 (2003), pp.203-210.

Hallaji, M., Seppänen, A. and Pour-Ghaz, M., Electrical resistance tomography to monitor unsaturated moisture flow in cementitious materials, Cement and Concrete Research, Vol. 69 (2015), pp.10-18.

Hiratsuka, A. and Kyu, I., A technique for evaluating solid/liquid separation in sludge as viewed from both quantity and quality of the separated liquid, Environmental System Research, Vol.19 (1991), pp.59-69.

Krieger, I. M. and Dougherty, T. J., A mechanishm for non-Newtonian flow in suspensions of rigid spheres, Transactions of The Society of Rheology, Vol.3 (1959), pp.137-152.

Lutran, P. G., Ka, M. Ng. and Delikat, E. P., Liquid distribution in trickle-beds. An experimental study using computerassisted tomography, Industrial \& Engineering Chemistry Research, Vol.30, No.6 (1991), pp.1270-1280.

Ogawa, T., Tadokoro, M. and Ohno, S., Characteristics of solid-liquid separation of waster excess sludge from smallscale on-site domestic wastewater treatment systems, Journal of the Japan Society of Waste Management Experts, Vol.7, No.1 (1996), pp.1-7.

Rintoul, M. D. and Torquato, S., Computer simulations of dense hard-sphere systems, The Journal of Chemical Physics, Vol. 105, No.20 (1996), pp.9258.

Tan, C., Wang, N. and Dong, F., Oil-water two-phase flow pattern analysis with ERT based measurement and multivariate maximum Lyapunov exponent, Journal of Central South University, Vol.23, No.1 (2016), pp.240-248.

Wang, P., Baolong, G. and Nan, L., Multi-index optimization design for electrical resistance tomography sensor, Measurement: Journal of the International Measurement Confederation, Vol.46, No.8 (2013), pp.2845-2853.

Wildemuth, C. R. and Williams, M. C., Viscosity of suspensions modeled with shear-dependent maximum packing fraction, Rheologica Acta, Vol. 23, No.6 (1984), pp.627-635.

Zhao, T., Eda, T., Achyut, S., Haruta, J., Nishio, M. and Takei, M., Investigation of pulsing flow regime transition and pulse characteristics in trickle-bed reactor by electrical resistance tomography, Chemical Engineering Science, Vol.130 (2015), pp.8-17.

Zhao, T., Iso, Y., Ikeda, R., Okawa, K. and Takei, M., Real-time measurement of particle volume fraction in centrifugal fields by wireless electrical resistance detector, Flow Measurement and Instrumentation, Vol.65 (2019), pp.90-97. 